\title{
TRATAMIENTO DE LAS QUEMADURAS EN LOS NIFOS
}

\author{
Por el Dr. RENE ARTIGAS NAMBRARD
}

Servicio de Cirugía Infantíl, Ortopedia y Traumatologia del Dr. A. 'Inostrosa y Posta Infantil de Primeros Auxilios - Hospital Manuel Arriarán

Fn esta ocasión analizaremos nuestra experiencia en el tratamiento de las quemaduras en los niños.

Bosquejaremos en primer lugar en líneas generales, la estadística pertinente que abarca los últimos diez años. Con este objeto bemos analizado el número total de enfermos que ha consultado en la Posta Infantil, siendo hospitalizados los que tenían una superficie quemada superior a un $20 \%$ y los inferiores a esta cifra si la quemadura comprometia la cara; los restantes enviados a su domicilio y posteriormente controlados en la Policlínica de Cirugía.

$$
\text { Cuadro } N^{*} 1
$$

Estadistica general de atcnciones por quemaduras durante los ultimos diez años

\begin{tabular}{cccc}
$\begin{array}{c}\text { N aten- } \\
\text { ciones Posta } \\
\text { Infantil }\end{array}$ & $\begin{array}{c}\text { Hospita- } \\
\text { lizados }\end{array}$ & $\begin{array}{c}\text { Falle- } \\
\text { cidos }\end{array}$ & $\begin{array}{c}\text { Porcentaje } \\
\text { mortalidad }\end{array}$ \\
\hline
\end{tabular}

\begin{tabular}{llllr}
\hline 1942 & 192 & 32 & 13 & $40,6 \%$ \\
1943 & 266 & 63 & 17 & $27,03 \% \%$ \\
1944 & 340 & 86 & 14 & $16,27 \%$ \\
1945 & 353 & 76 & 10 & $13,15 \%$ \\
1946 & 361 & 76 & 12 & $15,78 \%$ \\
1947 & 320 & 51 & 5 & $9,8 \%$ \\
1948 & 390 & $7 \mathrm{I} \%$ & 4 & $5,0 \%$ \\
1949 & 404 & 63 & 8 & $12,69 \%$ \\
1950 & 480 & 54 & 4 & $7,4 \%$ \\
1951 & 515 & 71 & 10 & $14,08 \%$ \\
\hline
\end{tabular}

Vemos en este cuadro que el número de enfermos que consultan ha ido aumentando progresivamente con los años, en cambio, el número de hospitalizados ha sufrido variaciones relativamente escasas, lo que se debe a que nuestro Servicio de Urgencia es cada vez más conocido y actualmente se recurre a él en forma sistemática y no sólo en los casos graves como en sus comienzos.

El porcentaje general de fallecimientos ha experimentado un brusco descenso, que se hace muy evidente desde 1945, fecha que coincide con el cambio radical del plan de tratamiento de los quemados.
Creo innecesario relatar la gran varieđad de procedimientos utilizados anteriormente en el tratamiento de esta afección, cuyos resultados fueron casi siempre variables y dudosos.

$$
\text { Cuadro No } 2
$$

Retacion entre la extension de la superficie quemada y la mortalidad. Sitio del follecimiento

\begin{tabular}{lccccc}
\hline & $20-50 \%$ & $55-70 \%$ & $75-100 \%$ & Posta & Servicio \\
\hline 1942 & 4 & 5 & 4 & 4 & 9 \\
1943 & 6 & 8 & 3 & 10 & 7 \\
1944 & 4 & 6 & 4 & 8 & 6 \\
1945 & 2 & 5 & 3 & 9 & 1 \\
1946 & 2 & 6 & 4 & 9 & 3 \\
1947 & 1 & 3 & 1 & 4 & 1 \\
1948 & 1 & 2 & 1 & 4 & 0 \\
1949 & 2 & 5 & 1 & 6 & 2 \\
1950 & 1 & 1 & 2 & 4 & 0 \\
1951 & 5 & 3 & 2 & 6 & 4 \\
\hline
\end{tabular}

Es interesante analizar la relación del porcentaje de fallecimientos con la extensión de la superficie corporal comprometida por la quemadura. En el cuadro $N^{9} 2$ podemos apreciar que hay una franca disminución de la mortalidad en quemaduras inferiores a un $50 \%$ a partir también del año 1945 , en cambio, en las extensiones superiores a esta cifra no hay ninguna variación, salvo la motivada por el distinto número de los ingresos.

Otro hecho de importancia es el lugar del fallecimiento de nuestros enfermos. La Posta Infantil es un sitio de hospitalización transitoria $y$ el enfermo es trasladado tan pronto como es posible al Servicio de Cirugía, donde se practican los tratamientos definitivos; por lo general, el enfermo no permanece más de 4 a 6 días en la Posta. Ahora bien, en el cuadro $N^{0} 2$ podemos apreciar que Ia mortalidad en el Servicio ha bajado a cero en dos oportunidades, siendo escasa en otros años, lo que viene a corroborar lo dicho anteriormente; la gravedad de los enfermos extensamente quemados es tal 
que fallecen en las primeras horas o días del accidente y no alcanzan a ser trasladados. Er cambio, el número de fallecidos en la Posta es muy similar en los diversos años.

Hace excepción a lo dicho anteriormente, lo ocurrido en el año 1951, año en que notamos un nuevo ascenso de la curva de mortalidad, cuyos motivos totalmente ajenos al plan de tratamiento, daremos más adelante.

Plan de tratamiento. Por regla general, el enfermo consulta por primera vez en la Posta Infantil e incluso dentro de las primeras horas del accidente, pese a lo cual ya ha sido atendido por su familia o amigos con los más variados procedimientos, siendo los más socorridos la salvadorina, harina cruda, bicarbonato de sodio, aceite de comer, e inclusn algunas veces tinta para escribir.

Desde su primera consulta en la Posta Inatil y posteriormente en el Servicio de Cigía, el enfermo es atendido por el mismo uipo médico, de modo que el plan terautico a que está sometido no sufre varia-

Cuadro No 8

\section{Parte General}

Calmar el dolor (morfina en solución, luminal, onema de cloraIy.

Cambatir el Schock. Plasma 200 cc. por cada lor de superficic quemada o 50 cc. por cada unidad sobre lo normal del hematocrito. Suero fisiokgico zota a gota despues. Oxígeno por sonda nasal.

Combatir la infección mediante antibióticos selecîisos.

Sucro antitetinico, si hay contaminación con tietra.

Régimen, hiperproteico.

Vitaminas. en especial C y complejo B.

Cortisona en dosis de 50 a 150 mgrs. según edad.

\section{Parte local}

Anestesia generpl segút cxtensión.

Suración reglada:

1) Aseo suero fisiologico tibio:

j) Eliminación flictenas rotas;

3) Tocación mercurio-cromo;

1) Pulverización sulfa-pcnicilina;

2) Aprisito graso ítull-grass);

Aposito corriente estéril:

7) Ventaje compresivo o ycsc, y

i) Cara al aire Jibre.

\section{Conducta posterior}

Suraciones espaciadas (cada 6 u 8 días).

Control hcmatológico seriado. COortegic anemia $\mathrm{y}$ saja hemoglobina).

Control proteinenuas. (Transfusioncs y amino-ácitos otales).

Zultivos superficic cruenta.

Vigilar actitudes $y$ posiciones.

injertos a delmátomo precoces. ciones, siendo, por lo tanto, su traslado de una parte a otra un mero accidente sin importancia.

Para mayor claridad, el plan de tratamiento Io hemos dividido en 3 partes, a saber: general, local y conducta posterior.

Iniciamos el tratamiento de nuestro paciente, calmándole el dolor, para to cual se usa luminal inyectable, enemas de cloral o morfina en soluciones especiales (un centigramo de morfina se diluye en 10 cc. de agua destilada), evitando de emplearla en niños menores.

Inmediatamente después se inicia la primera curación, si el estado del enfermo lo permite; en caso contrario es diferida dándole al schock toda nuestra atención. La curación bajo anestesia general, si el caso lo requiere, ya sea por la extensión comprometida o por el grado de excitabilidad del enfermo, la iniciamos con un prolijo lavado jabonoso con suero fisiológico tibio, de todos los bordes de la quemadura y sólo después las partes quemadas; eliminación sólo de las flictenas rotas y siempre que éstas no estén situadas en los pliegues de flexión de los dedos, tocación con mercurio-cromo, polvos de sulfa-penicilina y apósito graso (tullgrass), sobre el cual se colocan otras de gasa y algodón humedecidos y se termina con un vendaje compresivo, dentro de lo posible con vendas elásticas, y cuando la superficie quemada compromete los pliegues de flexión del codo o hueco poplíteo se practica vendaje enyesado.

Desde hace algún tiempo hemos abandarado por completo el método de curaciones al aire libre, menos en la cara; hemos visto que los enfermos así tratados tienen una evolución más larga, arrastrada y grave; la exudación de plasma es tan grande que el hematocrito se mantiene alto pese a las enormes cantidades de plasma que se inyecten, además el niño está siempre excitado, a pesar de los calmantes, lo que nos da la impresión que el dolor es mayor cuando la superficie quemada queda al descubierto; por otra parte, la superficie cruenta se cubre de una espesa costra adherente, que dempra en eliminarse y no es raro que bajo ella exista una colección purulenta, todo lo que retarda la cicatrización espontánea o la implantación de injertos.

Simultáneamente con la curación, o antes de proceder a ella, nos preocupamos del schock, usando para combatirlo, en primer 
lugar, el plasma gota a gota, a razón de $\mathbf{5 0 0}$ cc. por cada $10 \%$ de superficie quemada, continuando después con una mezcla de suero fisiológico con glucosado en la misma forma, líquidos azucarados por vía oral y una sonda nasal para la administración de oxígeno a permanencia.

Para prevenir futuras infecciones indicamos desde el primer momento penicilina $y$ estreptomicina, o bien una combinación de ambos antibióticos, los que serán reemplazados posteriormente de acuerdo con el resultado de los cultivos, cuando exista supuración; si las superficies cruentas están contaminadas con tierra se agrega suero antitetánico en dosis preventiva de $4.000 \mathrm{U}$.

Actualmente estamos en condiciones, pero no con la frecuencia que desearíamos, de usar cortisona, en dosis de 50 a 150 mgrs. diarios, según la edad de nuestros pacientes.

Tan pronto como es posible se indica un régimen hiperproteico, variando la forma de administración de acuerdo con la edad del niño, a lo que se agrega Vitamina $C$, Complejo B y Vitamina B.t.

Nuestra conducta posterior se adapta a cada caso en particular, pudiendo decir en líneas generales, que las curaciones las hacemos cada 6 u 8 días, dependiendo esto del estado de los apósitos, las sospechas de infección y la edad de los pacientes. Estas curaciones posteriores son hechas con la misma técnica y cuidados que la primera vez.

Es de rigor practicar controles bematológicos serados, dándole especial importancia a los valores del hematocrito, glóbulos rojos, porcentaje de hemoglobina y cantidad de proteínas; de acuerdo con los resultados se indica plasma, sueros, transfusiones de sangre total o aminoácidos. Para este estu: dio humoral contamos con la eficaz $y$ permanente colaboración del Servício de Transfusiones del Hospital.

Generalmente, después de la segunda curación, el enfermo es trasladado al Servicio de Cirugía, con el fin preconcebido de injertarlo tan pronto como sea posible, para lo cual se continúa con los mismos cuidados que anteriormente, vale decir, manteniendo el estado general con todos los medios a nuestro alcance y localmente practicando cultivos de la superficie cruenta para determinar la existencia, cantidad y calidad de gérmenes y luego administrar el o los antibiótícos eficaces.
Creemos que el injerto debe ser precoz en quemaduras de cierta profundidad, para evitar la cicatrización espontánea de los bordes, que determinará una cicatriz poco estética e irregular $y$, por otra parte, el riesgo que el injerto no adhiera bien en los bordes; es por esto que damos mayor importancia al estado de granulación de Ia superficie cruenta, que a una pequeña infección.

Usamos siempre los injertos tomados con el Dermátomo de Padgett de un espesor variable, entre 0,10 y $0,15 \mathrm{~mm}$. y los colocamos en block, suturándolos a los bordes con puntos separados, de bilo fino y a una tensión adecuada; si las superficies cruentas son muy extensas, practicamos injertos en estampillas, a pesar que hemos visto que los primeros prenden bien en un porcentaje cercano al $100 \%$ y dan una cicatriz lisa, uniforme de un aspecto muy satisfactorio; en cambio en los injertos en estampillas las cicatrices son irregulares, más pigmentadas $y$ de un resultado estético menos satisfactorio.

Suturado el injerto se espolvorea con sulfa penicilina $y$ sobre él se coloca un apósito graso seguido de otros algodonados y si se estima que la superficie de contacto entre zona cruenta e injerto no es satisfactoria, se agrega una delgada capa de esponja y se termina con un vendaje elástico.

Este mismo procedimiento usábamos para cubrir la superficie dadora, pero hemos cambiado de criterio, por cuanto eran frecuentes las hemorragias secundarias de esta zona $y$ las infecciones; estos inconverientes los hemos subsanado por completo, suprimiendo los apósitos grasos, los que son reemplazados por compresas humedecidas en suero o en una solución de protrombina.

La curación del injerto se levanta al quinto día, se practica aseo cuidadoso, se retiran los puntos $y$ se deja al aire libre con irrigaciones continuas o varias veces al día de una solución del antibiótico que se había demostrado eficaz.

La curación de la superficie dadora no se Ievanta hasta pasados 15 días, encontrándose por lo general ya totalmente cicatrizada.

Aquellos enfermos que tienen comprometida una superficie corporal reducida, son enviados a su domicilio y controlados posteriormente en la Policlínica del Servi- 
cio. En este tipo de enfermos se practican las curaciones con idéntico procedimiento, pero en este último tiempo, en los casos tratados personalmente, he usado el Picrato de Butesin en forma de ungüento en vez del tull-grass con espléndidos resultados, ya que el enfermo tiene una rápida sedación de su dolor $\mathrm{y}$ en las curaciones posteriores se aprecia una notable epitelización y raramente infección.

Este procedimiento no ha podido generalizarse por el costo del preparado y por la pérdida de tiempo que significa el preparar las gasas, inconveniente éste que podría subsanarse teniéndolas preparadas en cajas metálicas esterilizadas.

Resultados. Analizaremos a continuación los resultados obtenidos durante el año 1951, con estas normas de tratamiento (cuadro No 4).

\section{Cuadro No 4}

Material y resultados obtenitos en el tratamimio de 71 enfermos hospitalizados por quemadturas

A. Caracteristicas del material

\section{Edad}

Lactantes Niños

hasta $11 / 2$ a. mayores

$26 \quad 45$

B. Resultados

Favorables: 61

Extensión superficie quemada $20-50 \% \quad 55-70 \% \quad 75-100 \%$

66

66

2

Estada intrahospitalaria (clías)

$0-30 \quad 31.60$ 6L-100 +100

Fallccidos: 10 Epoca (dias)

$\begin{array}{llllllll}10 & 20 & 24 & 7 & 3 & 2 & .4 & 1\end{array}$

Consultaron en la Posta Infantil 505 enfermos, de Ios cuales se controlaron en la Policlínica 296 y se hospitalizaron 71, éstos tenían una superficie corporal comprometida superior a un $20 \%$. Como es lógico suponer, la mayor frecuencia de consultas se presentó en los meses de junio y julio: 78 y 66 enfermos, respectivamente.

Nos ocuparemos exclusivamente de los 71 enfermos hospitalizados, de ellos 35 eran hombres y $\mathbf{3 6}$ mujeres, y su edad fluctuó entre los 4 meses $\mathrm{y}$ los 12 an̂́os

La extensión de la superficie corporal comprometida fluctuó en la mayoría de los casos entre un 20 y $50 \%$ (66 enfermos), siendo felizmente muy pocos los que sobrepasaron estas cifras, sólo 5 , ya que estos últimos fallecieron todos.

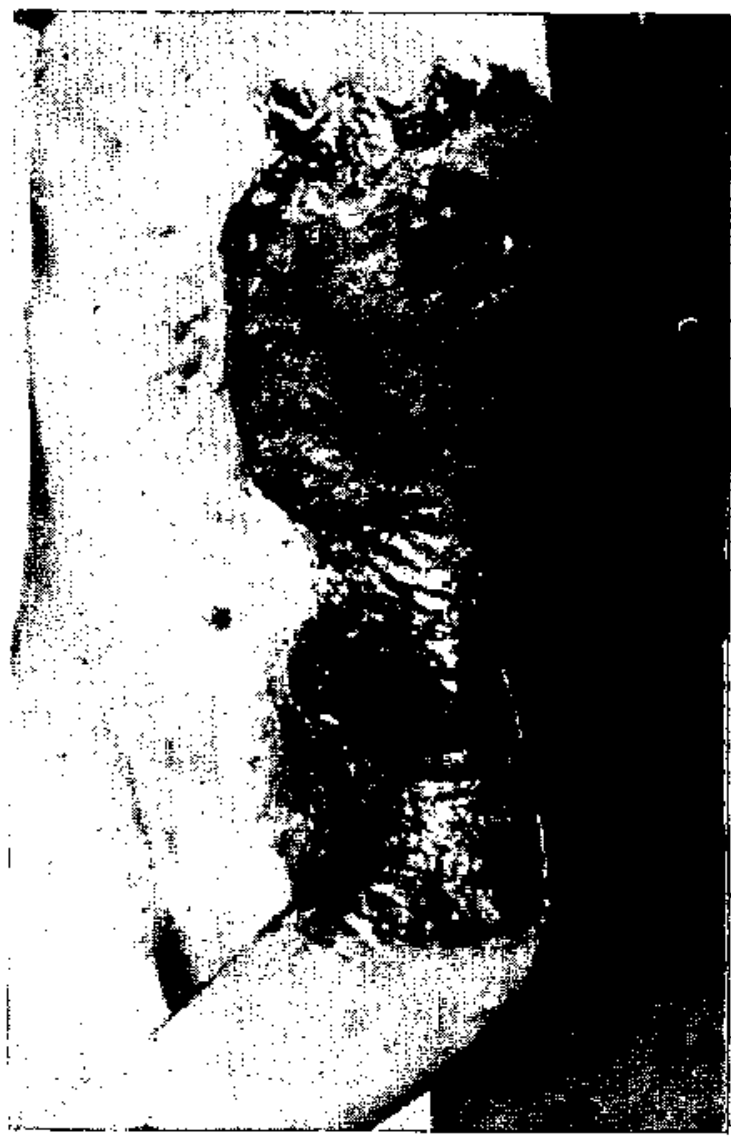

Foto 1

La curación espontánea de las lesiones se consiguió en un número reducido de enfermos (22) y se trató por lo general de quemaduras de $2^{\%}$ grado superficiales, que no se infectaron. En los 39 restantes fué necesario practicar injertos dermo-epidérmícos, de acuerdo con la técnica antes descrita. Para cubrir la totalidad de la superficie cruenta hubo necesidad en varias oportunidades de practicar injertos sucesivos; fué asi cómo bastó en sólo 10 casos con una toma de injertos para obtener la cicatrización, en cambio fueron necesarias 2 tomas en 19 enfermos, 3 en 6 y por último 4 tomas en 4 enfermos.

Obtuvimos un resultado favorable en 61 enfermos, o sea, $85,92 \%$, con una estada intrahospitalaria que fluctuó de los 17 días como mínimum y 246 días como máximum, siendo el promedio de estada entre los 35 y 100 días.

De estos 61 enfermos curados será necesario practicar correcciones posteriores en 


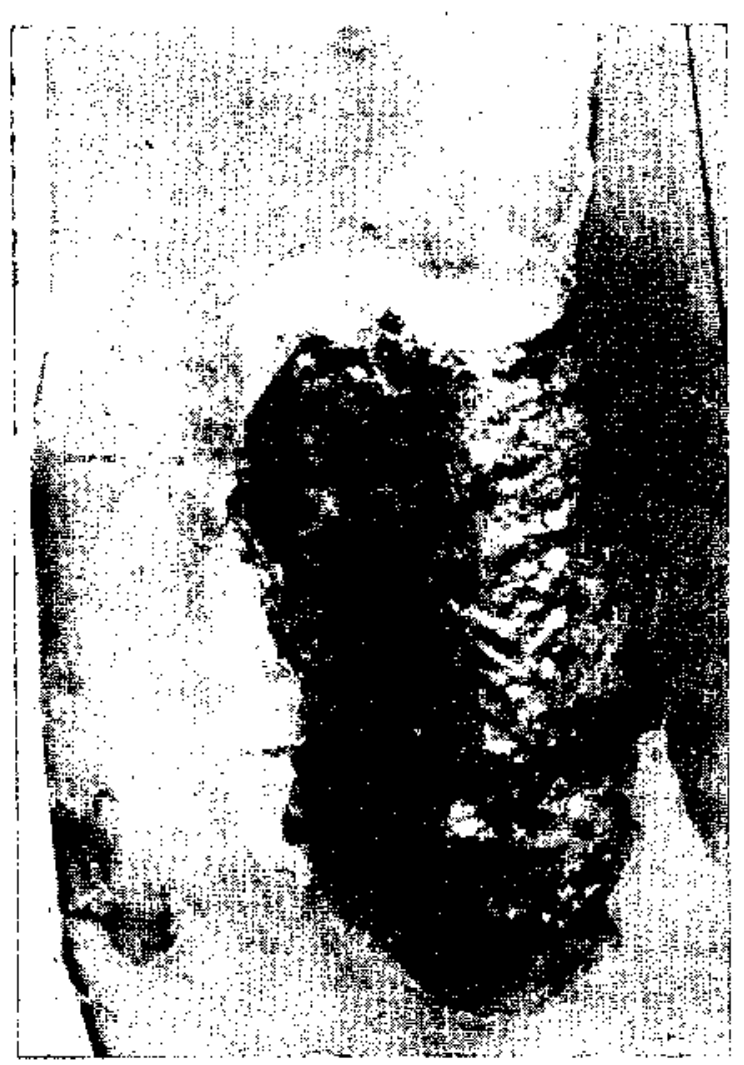

Foto 2

Fotos 1 y 2, Caso I.-Ingresa 7 meses después del accidente. Superficies cruentas supuradas $y$ mameJonadas.

5 de ellos, por las secuelas dejadas por las quemaduras, que han consistido en 3 casos en deformaciones faciales: ectropión de los párpados o alteraciones bucales, el cuarto por posición viciosa, los ortejos izquierdos y el quinto por deformación de la mano.

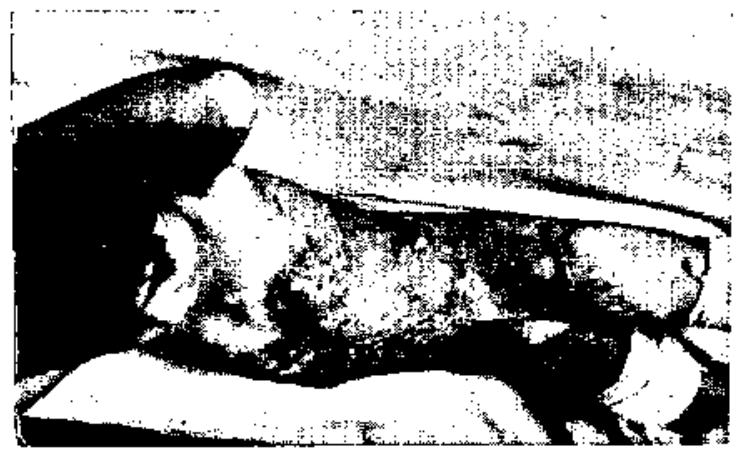

Foto 3
Los 10 enfermos restantes fallecieron, 5 de ellos en las primeras 48 horas de ocurrido el accidente, $\mathrm{y}$ todos ellos presentaban lesiones muy extensas y severas, superiores a un $50 \%$ de la superficie corporal. Los otros 5 fallecieron en los días sucesivos $y$ sus lesiones eran de mucho menor gravedad y la causa de su muerte fué en todos ellos procesos pulmonares agudos, que siguieron un curso fatal, pese a los antibióticos.

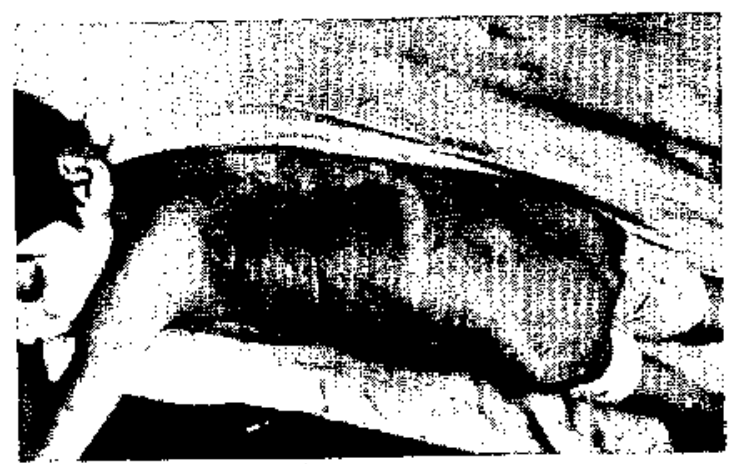

Foto 4

Fotos 3 y 4, Caso 1.-Después de 3 aplicaciones de injectos en block. Cicatrización total, a los 103 días de su ingreso.

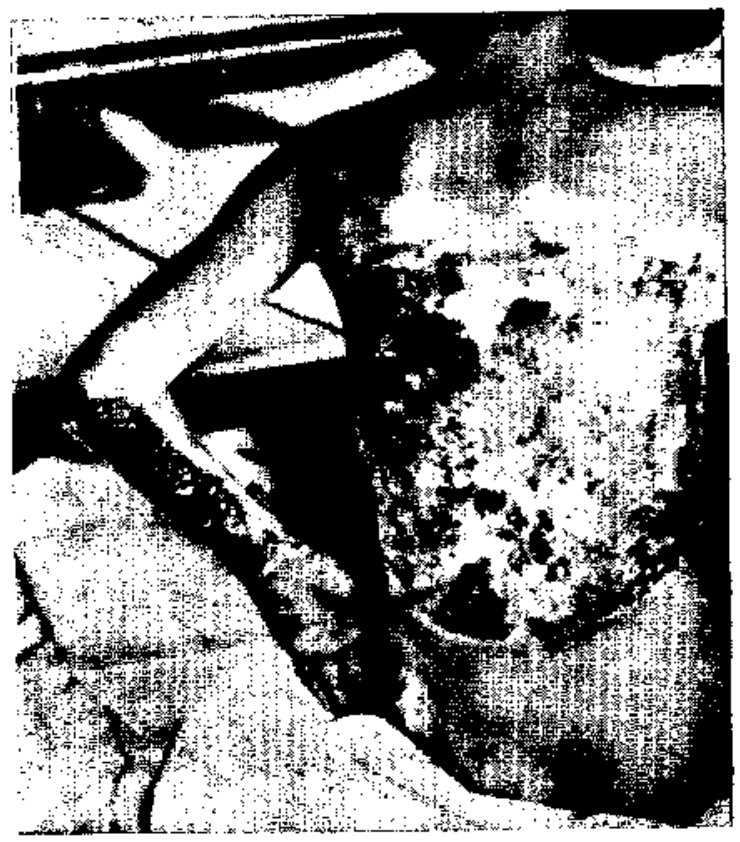

Foto 5, Caso II-Extensas quemaduras por fuego. In. gresó a las 6 horas del accidente. 


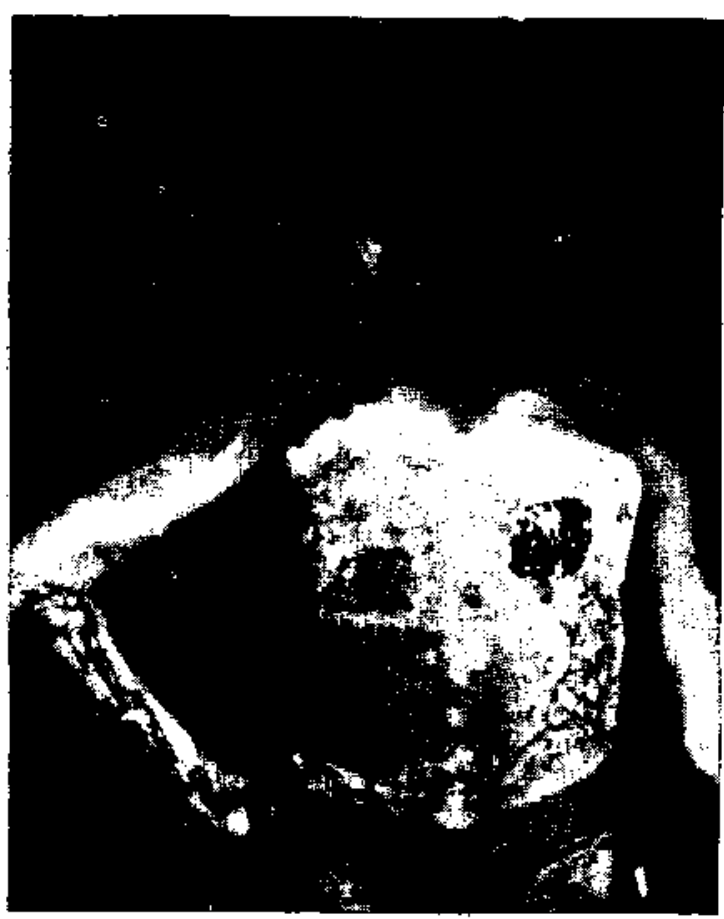

Foto 6

Foto 6, Caso 1I-Después de 3 aplicaciones de injertos, en block y estampillas. La zona cruenta residual se injertó de nuevo. En las zonas glúteas se aprecia parte de la superficic dadora, cicatrizada.

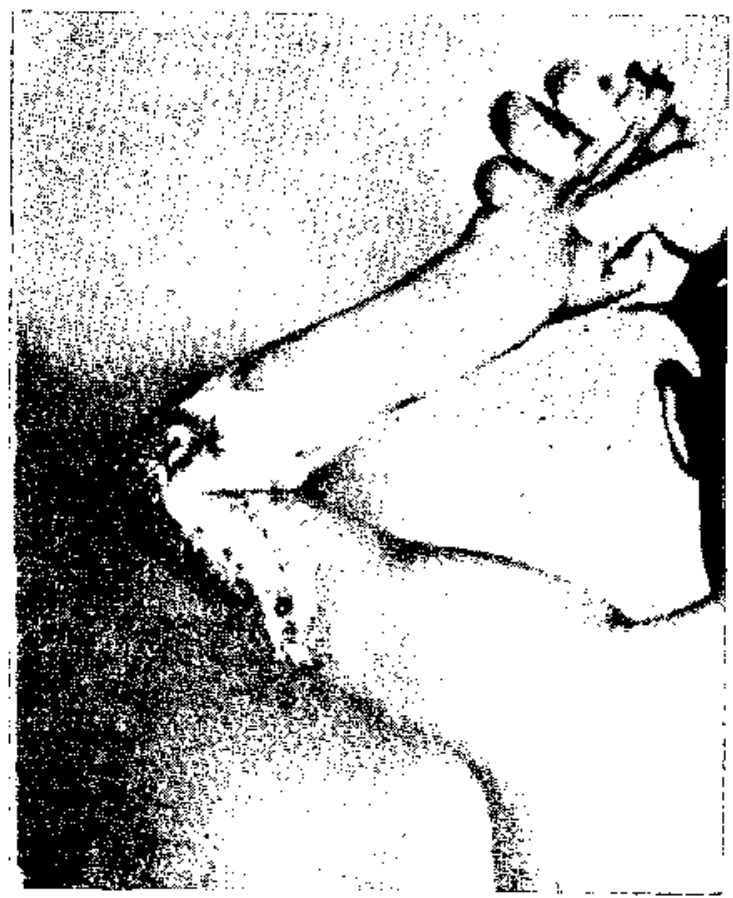

Foto 7
Cortisona. Desde diciembre de 1951 hasta la fecha hemos tenido oportunidad de tratar 8 enfermos quemados con cortisona. Su número tan restringido y su tan reciente aplicación no nos permiten sacar conclusiones definitivas, motivo por el cual nos limitaremos a exponer algunos casos.

Estimarnos que debe usarse cortisona en quemados, especialmente en casos graves, por cuanto favorece la retención de cloro y sodio, que a su vez hace retener líquidos combatiendo, por lo tanto, el schock; inhi-

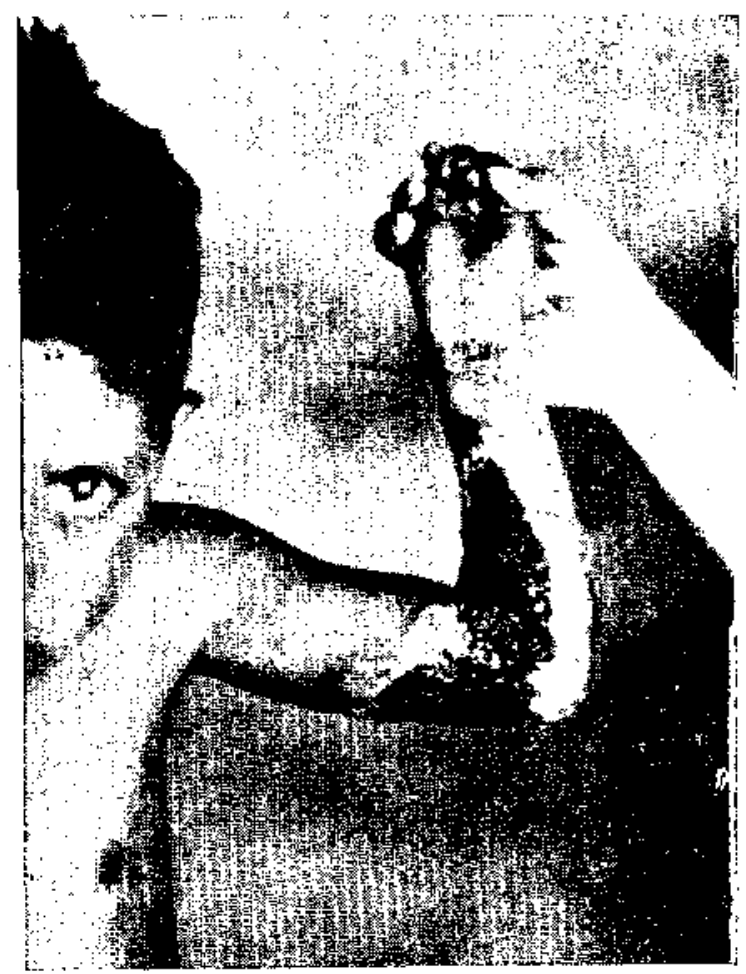

Foto 8

Fotos 7 y 8. Caso IIh-Quemaduras antiguas (45 dias). Codo en flexión superficies limpias.

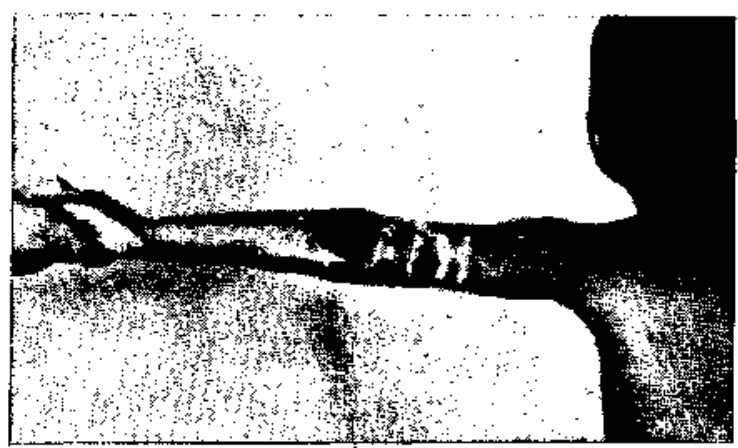

Foto 9 


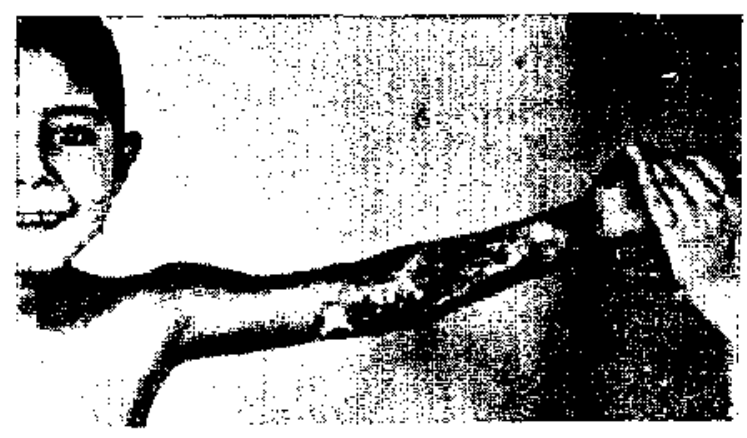

Foto 10

Fotos 9 y 10, Caso III.-Previa extensión del brazo se coloca yeso fenestrado. Injertos en block. Alta a los 30 días.

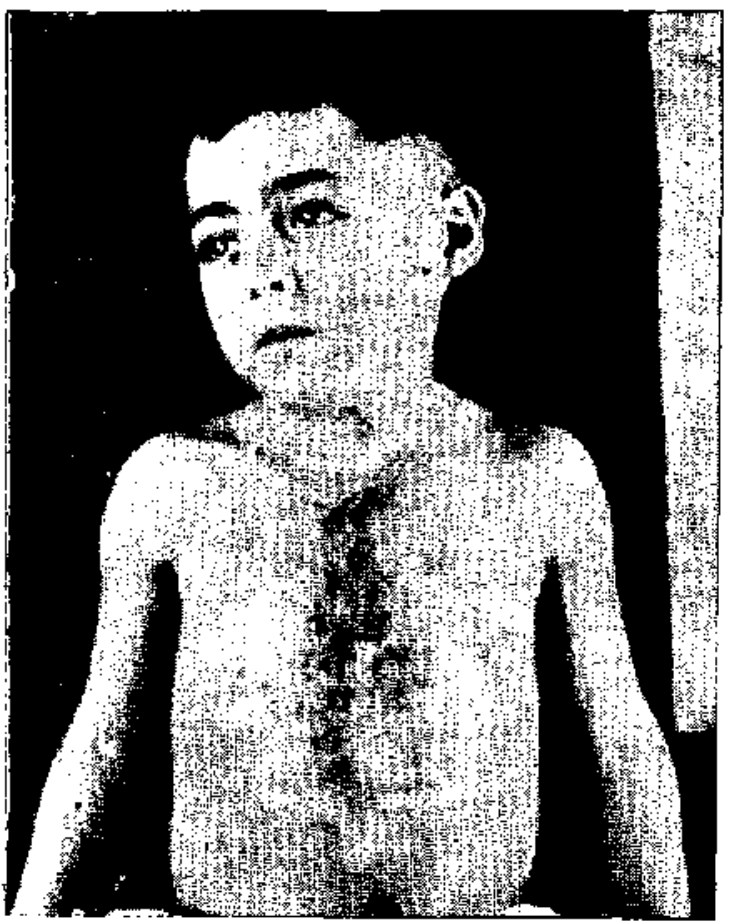

Foto 11, Caso IV.-Al final del tratamicnto. Injertos en estampillas.

be la excesiva proliferación de fibroblastos y favorecería la epitelización, lo que es francamente espectacular en quemaduras de la cómea (casos 4 y 6). Tendría, además, cierta acción anestésica, antipirética y antiinflamatoria y por último, detiene la actividad del tejido conjuntivo, evitando, por lo tanto, en cierto grado la aparición de retracciones cicatriciales.

De acuerdo con la experiencia de autores extranjeros, cuando usamos la cortiso- na cuidamos una serie de medidas dietéticas y controles seriados de laboratorio para evitar complicaciones de importancia: régimen hiperproteico, pobre en sal, control de líquidos ingeridos $\mathrm{y}$ eliminados, vigilancia de la actitud psiquica del enfermo, ánimo y grado de euforia. Controles seriados de presión, recuento de glóbulos rojos y eosinófilos, potasemia y reserva alcalina.

Respecto a las dosis las usamos bajas, 50 a 150 mgrs. diarios y mantenidas pocos días, los necesarios para que el enfermo esté fuera de todo peligro de schock y posteriormente reiniciamos el tratamiento unos 2 ó 3 días antes de proceder a la colocación de injertos y manteniéndola unos 6 días después. Incluso en dos oportunidades, por escasez de droga usamos el colirio de cortisona local en la superficie cruenta que se preparaba para injertar. Los resultados obtenidos con el uso de la cortisona por vía parenteral son alentadores $y$ dignos de un mayor estudio.

Si analizamos el cuadro anterior podemos apreciar hechos que si tuvieran confirmación posterior serían de una importancia extraordinaria.

En primer lugar, en los casos 4 y 6 se trataba de quemaduras de la córnea con visión cero en uno de ellos y en el otro con apreciable pérdida de la visión, ambos fueron tratados con colirio de cortisona, una gota cada 5 minutos los dos primeros dias y posteriormente cada 15 minutos y más espaciadas después. Ambos enfermos recuperaron rápidamente la visión en forma total y completa, antes de los 10 días de tratamiento. En estos casos fuimos asesorados eficazmente por el especialista de nuestro Hospital.

En los 6 enfermos restantes, que por lo general, eran niños mayores, podemos apreciar que el agente causante de la quemadura era de gravedad (fuego, bencina, etc.) y las lesiones producidas muy extensas y profundas; pese a esto, tenemos actualmente 3 enfermos en tratamiento con una superficie corporal comprometida superior al $50 \%$, uno de los cuales lleva 56 días del accidente; esperamos de todos ellos una recuperación total, porque su estado general y el estado de sus superficies cruentas así lo indican.

Dos enfermos han fallecido (casos 7 y 8), pero especialmente en el último de ellos las lesiones eran tan extensas que consideramos 
un éxito la sobrevida de 23 días después del accidente.

Creemos que estamos en una etapa experimental en el uso de la cortisona, pero la observación cuidadosa de nuestros enfermos $\mathrm{y}$ un estusiasmo moderado en su uso, nos darán en breve plazo una norma segura para su aplicación.

$$
\text { Cu a dra N. } 5
$$

ANALISIS DE OCHO OBSERVACIONES DE QUEMADOS TRATADOS CON CORTISONA

\begin{tabular}{|c|c|c|c|c|c|c|c|c|}
\hline \multirow{2}{*}{ caso } & \multirow{2}{*}{$\begin{array}{c}\text { Nombre } \\
\text { Observac. } \\
\text { Edad }\end{array}$} & \multirow{2}{*}{$\begin{array}{c}\text { Agente } \\
\text { causante }\end{array}$} & \multirow[b]{2}{*}{ ZONAS AFECTADAS } & \multirow{2}{*}{$\begin{array}{c}\text { Porcen- } \\
\text { taje }\end{array}$} & \multicolumn{2}{|c|}{ CORTISONA } & \multirow{2}{*}{$\begin{array}{c}\text { Tiempo } \\
\text { Control } \\
\text { (dras) }\end{array}$} & \multirow{2}{*}{ Resultado } \\
\hline & & & & & D. diar. & D. total & & \\
\hline 1 & $\begin{array}{c}\text { C. S. C. } \\
\text { Part. } \\
8 \text { añns }\end{array}$ & Petardos & Tórax, abdomen m. inier. 2 y $3 \mathrm{gr}$. & $40 \%$ & 100 mst. & $1,9 \mathrm{gr}$. & 95 & Sano \\
\hline 2 & $\begin{array}{l}\text { I. A. C. } \\
5 \% .382 \\
4 \text { añog }\end{array}$ & Ague caljente & $\begin{array}{l}\text { Tórax, abdomen to. sup. e inf. } \\
\text { y } 3 \text { grado }\end{array}$ & $60 \%$ & 75 tngr. & 2,2 gt. & 56 & En trat. \\
\hline 3 & $\begin{array}{l}\text { P. R. V. } \\
104.236 \\
10 \text { años }\end{array}$ & Parafina & $\begin{array}{c}\text { Torax, abdomen m. ini. nalg. } \\
\text { gen. } 2 \text { y } 3 \text { gt. }\end{array}$ & $50 \%$ & $100 \mathrm{mgr}$. & $2,75 \mathrm{gr}$ & 23 & En trat. \\
\hline 4 & $\begin{array}{l}\text { M. C.E. } \\
103.369 \\
1 \text { a } 8 \mathrm{~m} .\end{array}$ & Agua caliente & $\begin{array}{c}\text { Cabexa, cara, cuelio, cornea } \\
2 \text { grado }\end{array}$ & $15 \%$ & $\overrightarrow{\mathrm{Co}}$ & & 60 & Sano ojos \\
\hline 5 & $\begin{array}{l}\text { M. B. L. } \\
104: 840 \\
10 \text { ång }\end{array}$ & Bencina & $\begin{array}{c}\text { Tكrax, abodonen m. sup. } e \text { inf. } \\
2 \text { y } 3 \text { grado }\end{array}$ & $60 \%$ & $150 \mathrm{mgr}$ & $1,15 \mathrm{gr}$. & 21 & En trat. \\
\hline 6 & $\begin{array}{l}\text { C. Z. } \\
98.545 \\
11 \text { afิos }\end{array}$ & Carbirro & Cara. córneas, 2 grado visión 0 & $10 \%$ & & & 29 & Sabo \\
\hline 7 & $\begin{array}{l}\text { R.I. S. } \\
103.901 \\
10 \text { añ }\end{array}$ & Incendio ropas & $\begin{array}{l}\text { Torax, abdoraen tn. inf.nalg. } \\
\text { gen. } 2 \text { y } 3 \text { gr. }\end{array}$ & $60 \%$ & 120 mgr. & 2,1 क्ष & 21 & Fallece \\
\hline 8 & $\begin{array}{l}\text { J. J. E. } \\
99.606\end{array}$ & Bencina & $\begin{array}{l}\text { T6rax, abdomen to. eup. e inf. } \\
\text { nalg. gen. } 2 \text { y } 3 \mathrm{gr} \text {. }\end{array}$ & $80 \%$ & $160 \mathrm{mgg}$. & $3,0 \mathrm{gr}$. & 23 & Fallece \\
\hline
\end{tabular}

\section{Resumen}

Se exponen los resultados generales obtenidos durante los últimos 10 años en el tratamiento de las quemaduras y se analizan 71 observaciones de enfermos hospitalizados en el año 1951. Finalmente se relatan 8 casos de quemados tratados con cortisona.

\section{Summary}

The general results obtained in the last 10 years in the treatment of burns are analyzed. 71 cases histories of patients hospitalized during the year 1951 are reviewed. 8 cases treated with cortisone are detailed.

\section{BIBI.IOGRAFIA}

1.-ALDUNATF, PH. E--Análisis crítico acerca de alganos tratamientos de urgencia en, quemaduras, heridas de los micmbros y de la cara. Arch.'Soc. Cirug. Ch, 3: IIl, 195l.

2.-BARRET, B. J--Rehabilitation of Patients with deeps Burns. J. A. M. A. 1405: 148, 1952.

3.-BLOCKER, G. T.-Local and General treatment of acute extensive burns. (The open-air regime). Lancet 499: 111, 1951.

4.-BROWN and MC DOWELL--Skin Grafting of
Burns. Ed. Lippincott. Philadelphia, 1948. Pág. 53.

5-GRAM, H. R.-The Management of Bums. Lancet 263: II, 1951 .

6.-EVANS, I. E. and col--Fluid and Electrolyte requirements in severe burns. An. Surg. 804: I35. 1952.

7.-HAMILTON, L. CH.-Treatment of Burns. Intcr. Coll. Sung. 354: XVU, 1952.

8.-MARIN, G. y JARA. O--Quemaduras. Arch. Scc. Ciruj. Ch, 1927: II, 1950.

9.-MARTIN, C. B. and coI.-The Effect of ACTH on Autogenous and Homologous Skin Grafts in Rabbits. Surgery 258: 31, 1952.

10.-MORGAN, A. J.-The influence of Cortisonc on the survival of Homografts of Skin in the Rabbit. Surgery 505: 30, 1951 .

11.-OSBORNE, P. R.-Burns: Initial trcatment Local and General. Brit. Med. Jour. 1025: 1950.

12.-QUISPE, O. A.-Injertos de piel en Quemarluras. Rcv. Hosp. Niño 7 : XIV, 1951 .

13.-SPRAGUE, R. and col.-Physiological effects of cortisone and pituitary adrenocorticotropic hormone (ACTH) in man. J. A. M. A. 1941: 144, 1950.

14.-WAILACE, B. A.-The exposure treatment of Bums. Lancet 501: 1951.

15.-WITELAW, M. J-Physiological reaction to Pituitary adrenocorticotropic hormone (ACTH) in severe burns. J. A. M. A. 85: 145, 1951.

16.-WRIGHT, L. $T$, and col-Aureomycin as an adjunct in the treatment of major burns. Surgery 769: 29, 1951 . 